\title{
Application of 'tying, binding and fixing operation' in surgical treatment of severe mixed hemorrhoids
}

\author{
HONG-XIANG HUANG ${ }^{1}$, YI-BO YAO ${ }^{2}$ and YING TANG $^{1}$ \\ ${ }^{1}$ Department of Anorectal Surgery, Xin Hua Hospital Affilliated to Shanghai Jiao Tong University \\ School of Medicine, Shanghai 200092; ${ }^{2}$ Department of Anorectal Surgery, Longhua Hospital, \\ Shanghai University of Traditional Chinese Medicine, Shanghai 200032, P.R. China
}

Received March 18, 2016; Accepted May 12, 2016

DOI: $10.3892 / e t m .2016 .3339$

\begin{abstract}
The aim of the present study was to examine the clinical value of 'tying, binding and fixing operation' in treating severe mixed hemorrhoids. A total of 160 patients with severe mixed hemorrhoids were selected and randomly divided into the experimental $(n=80)$ and control $(n=80)$ groups. The groups were treated using 'tying, binding and fixing operation' and Doppler ultrasound-guided hemorrhoidal artery ligation (DG-HAL), respectively. The results showed that the average operative time of the experimental group (35.57 \pm 6.17) was significantly higher than that of the control group (12.73 \pm 4.92$)$. There was no significant difference of blood loss during the operation between the two groups $(\mathrm{P}>0.05)$. There was also no significant difference in improving the hemorrhage symptom between the two groups $(\mathrm{P}>0.05)$. In addition, concerning improvement of prolapse symptoms and reduction of the volume of hemorrhoids, the experimental group were significantly improved as compared to the control group. No anal function damage in the two groups was identified, and the length of stay in hospital for the two groups was not significantly different $(P>0.05)$. However, the hospitalization cost in the experimental group $(5,334.77 \pm 875.54)$ was significantly lower than that of the control group $(8,551.81 \pm 1,806.54)$ and satisfaction degree was significantly higher than that of the control group. The incidences of perianal pain, anal edema and dysuria between two groups were not significantly different $(\mathrm{P}>0.05)$. There were 10 cases of secondary hemorrhage and 18 cases of infection in the experimental group, and 12 cases of secondary hemorrhage and 14 cases of infection in the control group, although the differences between the two groups were not statistically
\end{abstract}

Correspondence to: Dr Ying Tang, Department of Anorectal Surgery, Xin Hua Hospital Affilliated to Shanghai Jiao Tong University School of Medicine, 1665 Kongjiang Road, Shanghai 200092, P.R. China

E-mail: ying_tang1212@163.com

Key words: tying, binding and fixing operation, Doppler ultrasound-guided hemorrhoidal artery ligation, mixed hemorrhoid significant $(\mathrm{P}>0.05)$. The incidence rate of local hematoma in the experimental group (1.2\%) was significantly lower than that in the control group (15.0\%). The recurrence rate of the control group (22.5\%) was also significantly higher than that of the experimental group (2.5\%). In conclusion, tying, binding and fixing operation is a promising method that may be employed for the treatment of sever mixed hemorrhoids, and it is better than DG-HAL in improving the prolapse and reducing the volume of hemorrhoids.

\section{Introduction}

In the past, due to the limited understanding of hemorrhoid pathogenesis, freezing and laser therapy were usually applied to treat hemorrhoids, which have been gradually eliminated because of high expense and poor therapeutic effect (1). The rise of the anal cushion down hypothesis has led to greater understanding of hemorrhoid resulting in the use of novel operation methods (2). Previous investigations focused on the Milligan-Morgan operation, a procedure for prolapse and hemorrhoids (PPH), and Doppler ultrasound-guided hemorrhoidal arterial ligation (DG-HAL). The latter is a widely applied operation method in western countries $(3,4)$. The procedures of DG-HAL are comparatively safe, constituting this method as the most effective method for hemorrhoid (5). However, there are inevitable complications including postoperative secondary hemorrhage, infection, anal edge edema and pain, and the long-term effect remains to be clarified $(5,6)$.

At present, there are no uniform criteria for surgery selection for hemorrhoids. Clinicians therefore aim to improve surgical methods and develop a novel surgical operation method. 'Tying, binding and fixing operation' was suggested by Huang et al (7), which is the creative application based on the 'bound therapy' in Traditional Chinese Medicine (TCM). The method involves an operation based on the two etiological theories of varicosity and anal cushion down, which can not only improve varicosity but also restore and repair the anal cushions. The 'tying, binding and fixing operation' theory has been accepted to a certain extent, although sufficient clinical evidence is lacking.

In the current study, we selected DG-HAL, a widely applied and accepted method in western countries, as the control to compare the therapeutical effect of the TCM 'tying, binding 
and fixing operation' in treating hemorrhoids, post-operative complication, length of hospital stay and hospitalization cost. The therapeutical effect, feasibility and safety were also evaluated. The results indicated that this is a promising technique.

\section{Patients and methods}

Patients. A total of 160 patients with severe mixed hemorrhoids admitted to the Department of Anorectal Surgery, Xin Hua Hospital Affilliated to Shanghai Jiao Tong University School of Medicine (Shanghai, China) between June 2012 and June 2013 were selected. The digital rectal exam and anoscopy were used to evaluate the hemorrhage, prolapse and volume of internal hemorrhoid. Inclusion criteria for the study were: i) Age, 18-70 years; ii) patients conformed to the diagnostic criteria of mixed hemorrhoid $(8,9)$, and the internal hemorrhoid was degree III-IV; iii) the blood routine test, urine routine test, liver and kidney function examination were normal; and iv) there were no systemic diseases and other anorectal disease, such as anal fissure, anal fistula, and anorectal tumor. Patients conforming to the above criteria received information and education, which introduced the principle and advantages of DG-HAL and the 'tying, binding and fixing operation', the universality of DG-HAL and the innovativeness of the 'tying, binding and fixing operation' method. A total of 160 cases were voluntarily included in this clinical experiment, and all 160 patients provided signed informed consent for participation in the study. The 160 patients were randomly divided into the experimental group (tying, binding and fixing operation) and the control group (DG-HAL) with 80 cases in each group. The gender, age, degree of internal hemorrhoid and disease course were not statistically different $(\mathrm{P}>0.05)$ between the two groups, which were comparable (Table I).

Approval for the study was obtained from the ethics committee of Xin Hua Hospital.

\section{Operation method}

Preoperative preparation. Patients of the two groups were required not to consume a solid diet from 8:00 p.m. onwards. Prior to sleep, warm saline was used for cleansing enema, and the morning that surgery was performed, patients received enema of warm saline. The lumbar anesthesia was used for anesthesia, and the position was lithotomy position.

Experimental group. The anus was disinfected and sufficiently dilated, and when the finger touched the hemorrhoid artery, the 2-0 absorbable suture of 5/8 radian was used for transfixion, and the prolapsed hemorrhoid was compressed and pushed to the anatomic site. The needle was entered from 0.3 to $0.5 \mathrm{~cm}$ above the dentate line, and exited the hemorrhoids from the other side to make an ' 8 ' suture. The hemorrhoids were pushed and compressed, and the ' 8 ' suture was made at $1.3-1.5 \mathrm{~cm}$ above the dentate line. For larger hemorrhoids, the ' 8 ' suture was made at $2.3-2.5 \mathrm{~cm}$ above the dentate line. Following transfixion, pushing, compression and transfixion, the prolapsed hemorrhoids were completely fixed above the dentate line.

Control group. After the anus was disinfected and dilated, a Doppler ultrasound anoscope and probe (AMI Co., Feldkirch, Austria) were inserted. When the probe was inserted to
2.0-3.0 $\mathrm{cm}$ above the dentate line, it was rotated along the vertical axis of rectum and anus, and the sound from the Doppler detector and the arterial signal on the screen were observed. After finding the artery, 2-0 absorption suture was used for the ' 8 ' suture. The anoscope was rotated by $360^{\circ}$ to ligate the hemorrhoidal arteries, after which the probe was rotated again to examine the ligation effect. After the hemorrhoidal arteries were examined, the probe and anoscope were removed.

Postoperative management. The patients in the two groups received intestinal mucosa protector (titanoreine suppository), and the dressing was changed in the morning and evening or after defecation daily. Following the operation, cefuroxime was intravenously injected for 48-72 h to prevent the infection. Following the operation, the patients were requested to remain in bed, and had liquid food on the same day. Patients followed a normal diet after $24 \mathrm{~h}$.

Drugs and equipments. Titanoreine suppository, generic name: Compound Carraghenates Suppositories (Xian-Janssen Pharmaceutical Co., Ltd., Xian, China). Cefuroxime sodium for injection was purchased from Beijing Pharmaceutical Co., Ltd. (Beijing, China). HAL-Doppler II Doppler diagnostic apparatus and the matched anoscope were purchased from AMI.

Observational indicators and follow-up. Observational indicators for the study were: i) Conditions during the operation, the operative time, the intraoperative blood loss; ii) evaluation indicators of therapeutic effect: hemorrhage, prolapse improvement, and shrinkage of hemorrhoid volume, the preservation of anal function and satisfaction degree survey; iii) length of stay and hospitalization cost; iv) postoperative complications: secondary hemorrhage, infection, thrombogenesis, anal enema, pain and urination; and v) 1-year reoccurrence rate.

The patients were observed for 7-10 days after surgery. After 7-10 days of surgery, the anus surrounding was inspected. The patient did not undergo digital rectal examination to avoid tearing of the suture. At 1 month following surgery, the patient underwent digital rectal examination and an anoscope was used to identify hemorrhage, prolapse and hemorrhoids, which were compared with pre-operative conditions to observe improvement of the symptoms in order to evaluate the therapeutic effect. The condition of anal function preservation was recorded to survey the satisfaction degree of the therapeutic effect. The postoperative complications of patients after 3 months were recorded, the pain and urination on the 1st day were evaluated, the anal enema condition on the 3rd day was evaluated (pain mostly occurs on the 1st day, while an enema mostly occurs on the 3 rd day). The reoccurrence condition of the hemorrhoid was followed up, and hemorrhoids that conformed to the diagnosis and treatment of hemorrhoids were diagnosed as reoccurrence.

\section{Evaluation criteria}

Operative effect. The effect was evaluated according to the hemorrhage, improvement of prolapse, and shrinkage of hemorrhoid volume: i) Recover indicated $>90 \%$ symptoms or physical symptoms disappear; ii) effective indicated 
Table I. General clinical data for two groups.

\begin{tabular}{|c|c|c|c|c|}
\hline Item & Experimental group $(n=80)$ & Control group $(n=80)$ & $\mathrm{t} / \chi^{2}$ value & P-value \\
\hline Gender, male/female & $43 / 37$ & $47 / 33$ & 5.327 & 1.518 \\
\hline Age, year & $53.16 \pm 12.16$ & $51.74 \pm 12.63$ & 2.736 & 3.527 \\
\hline Degree of internal heamorrhoids, case & & & 6.724 & 1.196 \\
\hline III & 55 & 51 & & \\
\hline IV & 25 & 29 & & \\
\hline Disease course, month & $84.49 \pm 101.56$ & $95.16 \pm 107.75$ & 4.629 & 2.117 \\
\hline RBC count, $\times 10^{12} / 1$ & 4.6 & 4.5 & & \\
\hline Hb count, g/1 & 98 & 100 & & \\
\hline
\end{tabular}

RBC, red blood cell; Hb, hemoglobin.

$>50 \%$ symptoms or physical signs disappear; and iii) invalid indicated $<50 \%$ symptoms and physical signs disappear.

Determination of postoperative pain, enema and urinary retention. Scoring criteria were: i) No indicated no pain of wound surface when defecation and changing dress, no enema of wound surface, and urination was fluent without discomfort; ii) mild indicated a slight discomfort when defecation and changing dress, the edema area was $\leq 1 / 4$ of anus surrounding area, and slight discomfort during urination; iii) moderate indicated pain on the wound surface that was alleviated when defecation and changing dress, patients were required to take analgesic, the edema area was $1 / 4-1 / 2$ of the anus surrounding area, and there was obvious difficulty during urination, albeit catheterization was not compulsory; and iv) severe indicated severe pain of wound surface, patients required to take strong analgesics such as dolantin or morphine, the edema area was $>1 / 2$ of anus surrounding area, and urination was difficult, requiring catheterization.

Statistical analysis. SPSS 19.1 software (IBM SPSS, Armonk, NY, USA) was used to analyze the data. Quantitative data, such as age, disease course and operative time, were presented as mean \pm standard deviation, and the t-test was used for comparison among groups. Enumerated data such as gender, stage and some postoperative complications, were analyzed using the $\chi^{2}$ test. Data in the two groups were classified as: therapeutic effect, satisfaction degree and some postoperative complications; these were analyzed by Wilcoxon rank-sum test. $\mathrm{P}<0.05$ was considered to indicate a statistically significant difference.

\section{Results}

Analysis of typical case. Analysis of typical cases in the two groups is shown in Figs. 1 and 2.

The experimental group included a 58-year-old male patient with complication of anal skin tag for 10 years. The anal lump prolapsed for 3 years, and could be returned spontaneously or by hand to the anal canal. In the last 2 weeks the symptoms were aggravated, the blood in stool was increased showing fresh red color and had a teardrop shape, the prolapsed tissue could not be returned spontaneously or by hand to the anal canal. A physical examination showed that there was dark violet circularity goiter, edema of skin surface, with hard texture and pressing pain. There was prolapse of internal hemorrhoids, which could be returned by hand. Anoscopy examination showed that the volume of hemorrhoids was $\sim 1.2 \mathrm{~cm}^{3}$, there was mucous membrane erosion at points 5 and 7 . The blood routine test showed red blood cells (RBC) $3.50 \times 10^{12} / 1$, and hemoglobin (Hb) $10^{9} \mathrm{~g} / 1$. The diagnosis was severe mixed haemorrhoids, the degree of hemorrhoids was degree IV, and there was slight anemia. There was no severe disease history in the past. Patients received 'tying, binding and fixing operation' at the Xin Hua Hospital Affilliated to Shanghai Jiao Tong University School of Medicine (Fig. 1), the operation duration time was $\sim 45 \mathrm{~min}$, the intraoperative blood loss was $\sim 8.5 \mathrm{ml}$, there was no obvious hemorrhage in stool and no hemorrhoid prolapse at 3 days after operation, and the pain was alleviated. The patient was discharged on the 4th day and the hospitalization cost was $\sim 700$ dollars. In the follow up, the hemorrhage and prolapse symptoms disappeared, the hemorrhoids were almost disappeared, there was no reoccurrence within 1 year and no adverse effect on defecation. The blood routine test was recovered and the patient was satisfied with the therapeutic effect.

The control group included a 62-year-old female, with complication of hematochezia for 10 years. The anal lump prolapsed for 5 years, but could be returned spontaneously and by hand to the anal canal. In the last 1 month, the symptoms were aggravated, the prolapsed tissue could not be returned with intolerable pain. Physical examination showed a dark violet mass around the anus, and prolapse of internal hemorrhoids and hemorrhage on the surface were obvious. The texture was hard and there was compressing pain. There were $\geq 3$ hemorrhoids that could not be returned by hand to the anal canal. The anoscopy examination showed that there was diffuse erosion and hemorrhage on the surface of hemorrhoids, and the hemorrhoids volume was $2.6 \mathrm{~cm}^{3}$. The blood routine test showed $\mathrm{RBC} 3.0 \times 10^{12} / 1$, and $\mathrm{Hb} 88 \mathrm{~g} / 1$. The patient was diagnosed as having severe mixed hemorrhoids, the internal hemorrhoids degree was degree IV, and had moderate anemia. The patient had no severe disease history, and underwent DG-HAL surgery (Fig. 2), with an operation time of $20 \mathrm{~min}$. Blood loss during operation was 

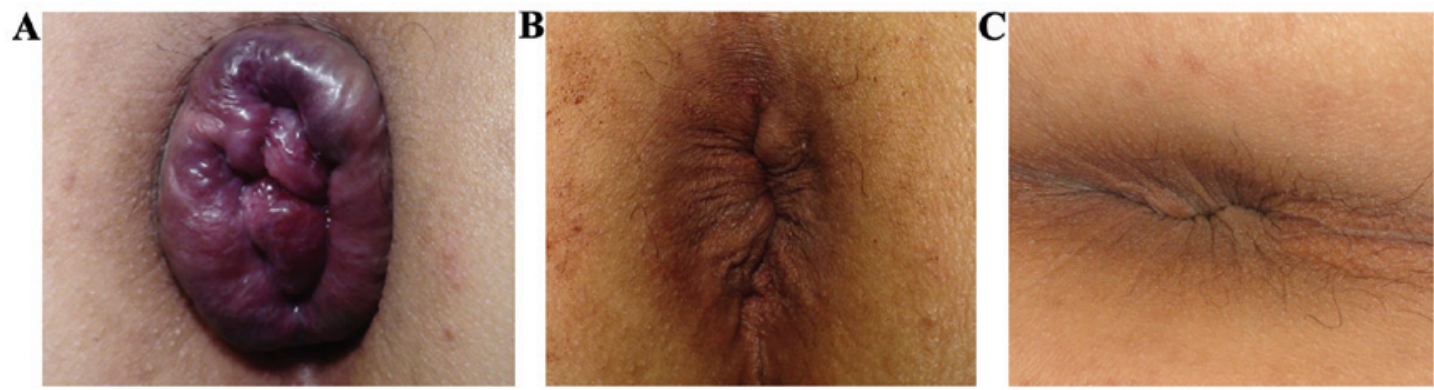

Figure 1. Therapeutic effect of 'tying, binding and fixing operation'. (A) Male, 58 years of age exhibiting severe mixed hemorrhoids. Internal hemorrhoids were degree IV, the proplapse symptom was obvious, the volume of hemorrhoids was $\sim 1.2 \mathrm{~cm}^{3}$. (B) At 7 days after the operation, the internal hemorrhoids were returned without proplapse or hemorrhage, and there was slight varicosity on the external hemorrhoids. (C) At 1 month after the operation, the prolapsed symptom disappeared and the hemorrhoids almost disappeared, and the varicosity of external hemorrhoids was improved without secondary hemorrhage, infection or local hematoma.
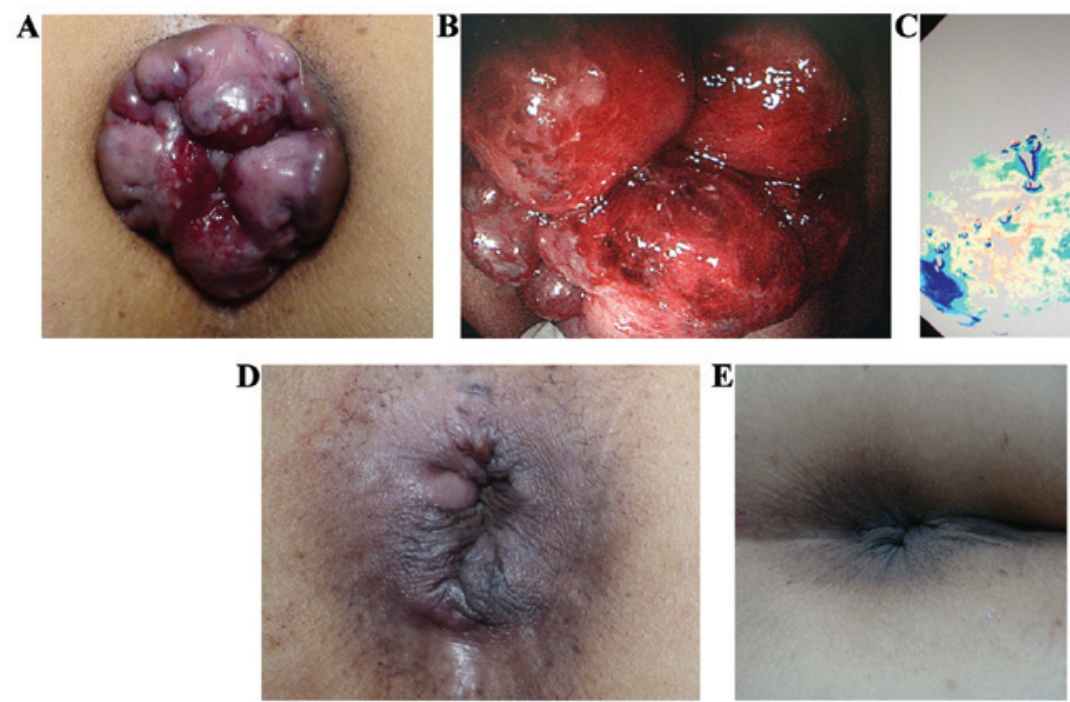

Figure 2. Therapeutic effect of DG-HAL. (A) Female, 62 years of age exhibiting severe mixed hemorrhoids. Internal hemorrhoids were degree V, there were obvious hemorrhage and prolapse symptoms, and the volume of hemorrhoids was $2.6 \mathrm{~cm}^{3}$. (B) Anoscope examination, there were $\geq 3$ hemorrhoids, the total volume was around $2.6 \mathrm{~cm}^{3}$, and there was diffuse erosion and hemorrhage on the surface. (C) Hemorrhoidal artery can be accurately and rapidly localized by the signal on the screen of Doppler ultrasound equipment. (D) At 8 days after the operation, hemorrhage and prolapsed symptoms disappeared, there was moderate varicosity on external hemorrhoid, and the hemorrhoid was invisible. (E) At 1 month after operation, there was no hemorrhage, the prolapsed and varicosity of external hemorrhoidal varicosity were improved with slight skin tag, and there was no secondary hemorrhage or infection.

$\sim 7.5 \mathrm{ml}$, the prolapse symptoms disappeared at 3 days after the operation, although there was obvious edema around anus, a little blood and obvious pain when defecation. After 8 days of operation, the prolapse symptom disappeared and there was moderate varicosity on external hemorrhoids after which the patient was discharged. The total hospitalization cost was $\sim 1,400$ dollars. After 1 month follow up, there was no hemorrhage or prolapse symptom, the external varicosity was improved, and there was only slight skin tag without secondary hemorrhage or infection. There was no reoccurrence within 1 year and no dysporia, the anemia was improved and the patient was satisfied.

Conditions during operation and operative effect. In the comparison of conditions during operation, the average operative time of the experimental group $(35.57 \pm 6.17)$ was significantly higher than that of the control group $(12.73 \pm 4.92)$. There was no significant difference in the intraoperative blood loss between the two groups $(\mathrm{P}>0.05)$ (Table II).
The therapeutic effect showed no significant difference in improving hemorrhage symptoms of the two groups $(\mathrm{P}>0.05)$. However, regarding improvement of prolapse symptoms and reduction of the volume of hemorrhoids, the experimental group was significantly better than the control group. There was no anal function damage in the two groups (Table III). The 1-year reoccurrence rate $(22.5 \%)$ in the control group was significantly higher than that in the experimental group $(2.5 \%)$ (Table III).

Postoperative complications. Postoperative complications were followed up, perianal pain and urination were observed on the 1st day, anal edema was observed on the 3rd day, and the other complications were observed within 3 months. The incidences of perianal pain, anal edema and dysuria between the two groups were not significantly different $(P>0.05)$, and $<7 \%$ patients were severe. The above complications disappeared spontaneously or following treatment (Table IV). There were 10 cases of secondary bleeding and 18 cases of infection 
Table II. Comparison of the conditions in operation.

\begin{tabular}{|c|c|c|c|c|}
\hline Conditions in operation & Experimental $(n=80)$ & Control group $(n=80)$ & T-value & P-value \\
\hline Operation duration time, min & $35.57 \pm 6.17$ & $12.73 \pm 4.92$ & 13.693 & 0.000 \\
\hline Blood loss, ml & $8.52 \pm 4.61$ & $6.35 \pm 3.28$ & 0.713 & 8.652 \\
\hline
\end{tabular}

Table III. Comparison of operative efficacy and length of stay between the two groups.

\begin{tabular}{|c|c|c|c|c|}
\hline Item & Experimental group $(n=80)$ & Control group (n-80) & $\mathrm{t} / \chi^{2} / \mathrm{T}$-value & P-value \\
\hline Hemorrhage symptom & & & $1.758^{\mathrm{a}}$ & 5.763 \\
\hline Recover & 38 & 35 & & \\
\hline Effective & 33 & 38 & & \\
\hline Invalid & 9 & 7 & & \\
\hline Prolapse & & & $11.976^{\mathrm{a}}$ & 0.008 \\
\hline Recovery & 47 & 24 & & \\
\hline Effective & 29 & 30 & & \\
\hline Invalid & 4 & 26 & & \\
\hline Shrinkage of hemorrhoid volume & & & $13.779^{\mathrm{a}}$ & 0.006 \\
\hline Recovery & 36 & 25 & & \\
\hline Effective & 34 & 22 & & \\
\hline Invalid & 10 & 33 & & \\
\hline Preservation of anal function & 80 & 80 & & \\
\hline Satisfaction degree & & & $9.751^{\mathrm{a}}$ & 0.001 \\
\hline Very satisfied & 16 & 7 & & \\
\hline Satisfied & 38 & 12 & & \\
\hline Ordinary & 22 & 46 & & \\
\hline Unsatisfied & 4 & 15 & & \\
\hline Length of stay & $5.18 \pm 1.79$ & $5.98 \pm 2.18$ & $4.217^{\mathrm{b}}$ & 0.985 \\
\hline Hospitalization cost & $5,334.77 \pm 875.54$ & $8,551.81 \pm 1,806.54$ & $8.436^{\mathrm{b}}$ & 0.025 \\
\hline Reoccurrence & 2 & 18 & $17.752^{c}$ & 0.000 \\
\hline
\end{tabular}

${ }^{a}$ Means T-value in Wilcoxon rank sum test; ${ }^{b}$ means t-value in t-test; ${ }^{c}$ means $\chi^{2}$ value in $\chi^{2}$ test.

Table IV. Comparison of postoperative complications between the two groups.

\begin{tabular}{|c|c|c|c|c|c|c|}
\hline $\begin{array}{l}\text { Postoperative } \\
\text { complications }\end{array}$ & $\begin{array}{l}\text { Perianal } \\
\text { pain }\end{array}$ & $\begin{array}{c}\text { Anal } \\
\text { edema }\end{array}$ & Dysuresia & $\begin{array}{l}\text { Secondary } \\
\text { hemorrhage }\end{array}$ & Infection & $\begin{array}{c}\text { Local } \\
\text { hematoma }\end{array}$ \\
\hline Experimental group $(n=80)$ & & & & 10 & 18 & 1 \\
\hline No & 37 & 33 & 30 & & & \\
\hline Mild & 32 & 32 & 35 & & & \\
\hline Moderate & 9 & 10 & 14 & & & \\
\hline Severe & 2 & 5 & 2 & & & \\
\hline Control group (n=80) & & & & 12 & 14 & 12 \\
\hline No & 40 & 35 & 32 & & & \\
\hline Mild & 30 & 31 & 26 & & & \\
\hline Moderate & 6 & 13 & 19 & & & \\
\hline Severe & 4 & 2 & 3 & & & \\
\hline$\chi^{2} /$ T-value & $8.754^{\mathrm{a}}$ & $6.579^{a}$ & $5.557^{\mathrm{a}}$ & $2.113^{\mathrm{b}}$ & $5.773^{\mathrm{b}}$ & $18.926^{\mathrm{b}}$ \\
\hline P-value & 0.857 & 1.372 & 1.569 & 6.187 & 1.897 & 0.000 \\
\hline
\end{tabular}

${ }^{\mathrm{a}}$ Means T-value in Wilcoxon rank sum test; ${ }^{\mathrm{b}}$ means $\chi^{2}$ value in $\chi^{2}$ test. 
in the experimental group, 12 cases of secondary hemorrhage and 14 cases of infection in the control group. However, the differences between the two groups were not statistically significant $(\mathrm{P}>0.05)$. Infection was alleviated spontaneously or following treatment, the incidence rate of local hematoma in the experimental group (1.2\%) was significantly lower than that in the control group (15.0\%) (Table IV).

Hospitalization cost. The length of hospital stay in the two groups was not significantly different $(\mathrm{P}>0.05)$. However, the hospitalization cost in the experimental group was significantly lower than that of the control group $(5,334.77 \pm 875.54$ vs. $8,551.81 \pm 1,806.54, \mathrm{P}=0.025)$, and the satisfaction degree was significantly higher than the control group (Table III).

\section{Discussion}

There is insufficient clinical practice criteria for the 'tying, binding and fixing operation' method to examine its potential therapeutic effect in severe mixed hemorrhoids and evaluate its clinical value. In the present study, we used DG-HAL, which is commonly applied and recognized in western countries, as the control to compare the effect in the surgical treatment of hemorrhoids, postoperative complication, length of stay and hospitalization cost, and assess the therapeutic effect and safety.

A comparison of the therapeutic effect in the two groups with regard to the 'tying, binding and fixing operation' method was significantly better than that of DG-HAL, mainly manifesting as improvement of prolapse symptoms and shrinkage of hemorrhoid volume. Previously, it was reported that in DG-HAL, hemorrhoidal arteries are ligated to effectively block the bleeding vessels, thus DG-HAL has a positive effect on hemorrhage symptoms (8). However, this method is not able to induce the degeneration of chronic hemorrhoid tissues and obvious prolapsed hemorrhoids cannot completely shrink following the establishment of collateral circulation, which leads to an increase in pressure of hemorrhoids tissue. Consequently, it is not good at improving prolapse symptoms and decreasing hemorrhoid volume (4-6,9-12). It has been reported that DG-HAL and PPH have good therapeutic effect, however, the effect for hemorrhoids with prolapse symptoms is poor, which demonstrated the limit of therapeutical treatment including DG-HAL $(13,14)$. In this study, the hemorrhoidal artery was also ligated in 'tying, binding and fixing operation' to decrease the pressure of hemorrhoidal tissue, which was similar to DG-HAL in improving hemorrhage symptoma. Additionally, during the operation the reseted anal cushion was ligated by absorbable suture, which was the foreign matter stimulus to cause local aseptic inflammation and gradual fibrosis of mucosa, and further repair the anal cushion at the normal anatomical position. This improves the symptom of prolapse; the creative point of this method was that the hemorrhoids were continuously compressed, pushed and ligated, which was the same as the TCM 'bound therapy' (15). The aim was to block the superficial hemorrhoidal vein to ensure deep blood circulation, which improves blood stasis and varicosity. There was also small thrombus formatted in the tissue between two sutures due to the long-term compression. Thus, the aseptic inflammation and suspension effect of the anal cushion were enhanced, which has a better effect in improving prolapse symptoms and reducing hemorrhage symptoms than the control group. The two groups had good effects in preserving anal function because the surgical trauma was little and the anal cushion tissue was not damaged, and suture and ligation were completed above the dentate line to avoid the stimulus to somatic nerve (16), thus its effect on defecation controlling was small. The reoccurrence rate of the control group was higher than that of the experimental groups, possibly due to the fact that although DG-HAL has good effect on hemostasis, the effect on improving hemorrhoid volume prolapse and reducing hemorrhoid volume was poor. Thus, the prolapsed tissue was not completely atrophic, and following the establishment of collateral circulation, the blood and pressure in hemorrhoid tissues were increased, and the risk of vein flexion and anal cushion moving down was increased (17). This led to the reoccurrence risk being increased and the satisfaction degree decreased. As DG-HAL was completed under the guidance of Doppler ultrasound, the hemorrhoidal artery was accurately located, which was significantly shortened the operative time.

However, due to the application of Doppler ultrasound equipment, the hospitalization cost was 600 dollars higher than the 'tying, binding and fixing operation' method, which may affect the independent choice of patients in the future.

Except the formation of local hematoma, the other complications were not statistically different between the two groups. The occurrence rates of perianal pain, uroschesis after 1 day in the two groups and the moderate and severe anal edema were $10-20 \%$, which may be improved spontaneously or after treatment, and there was no severe adverse event. The 'tying, binding and fixing operation' method and DG-HAL were minimally invasive operations, compared with the conventional Milligan-Morgan operation, the trauma was small and the operation was above the dentate line. Thus, the patients recovered rapidly and the length of stay was significantly reduced, theoretically the anal pain and discomfort caused by trauma was avoided $(4,18)$. However, practically because anal dilatation was not complete, it caused skin split or a clip was used to clip the perianal skin during operation; thus, postoperative perianal pain was inevitable. The anal edema was mainly considered to be associated with the operation, and $90 \%$ of the edema can disappear as recovery from the disease. The dysuresia was considered to be associated with lumbar anesthesia, and the pain stimulus to anus reflectively caused urethral sphincter muscle contraction to induce uroschesis (19). After observation, 95\% of uroschesis disappears with alleviation of pain. There was an infection rate and secondary hemorrhage in the two groups. Postoperative infection was considered to be due to the special infection susceptibility of anus (20), however, it can be controlled by antibiotics. The secondary hemorrhage manifested as sticky bloody stool, which was considered to be related to mucosa damage, ischemic inflammatory necrosis, constipation and suture tear caused by hard stool $(21,22)$. However, the hemorrhage was not severe, and it was suggested that patients at the Department of Anorectal Surgery following a light diet, with more fiber to avoid the secondary damage caused by constipation and hard stool. In the present study, $15 \%$ patients in the control group had local hematoma, however, there was no hematoma in the experimental group. The difference 
may be linked to the principles of the two methods. In the 'tying, binding and fixing operation' method, arterial blood can be prevented from entering the hemorrhoid tissue, and the venous blood can be extruded from the tissue to improve blood stasis. The absorbable suture is used to hang the hemorrhoids to rectum wall near to anus (7), which can improve the prolapse symptoms and effectively decrease the risk of local hematoma formation.

In the present study, 'tying, binding and fixing operation' is a promising method for the treatment of hemorrhoids, and is better than DG-HAL in improving the prolapse and reducing the volume of hemorrhoids. There were less postoperative complications, the anal function was not affected, the risk of hematoma formation and reoccurrence was significantly decreased, the postoperative satisfaction degree was improved and the hospitalization cost was significantly reduced.

Although there are many advantages, the 'tying, binding and fixing operation' may cause complications such as pain and secondary hemorrhage. As a novel method, there may be potential risks that have not been identified. There was an optimal effect in this study, however, this was just the primary step of 'tying, binding and fixing operation' application clinically. Future studies are therefore required to fully evaluate the clinical value of this method.

\section{Acknowledgements}

The present study was supported by Fund of Shanghai Sanitary Bureau (no. 2010L011A).

\section{References}

1. Higuero T: Hemorrhoids. Rev Prat 64: 867-874, 2014 (In French).

2. Henderson PK and Cash BD: Common anorectal conditions: Evaluation and treatment. Curr Gastroenterol Rep 16: 408, 2014.

3. Cerato MM, Cerato NL, Passos P, Treigue A and Damin DC: Surgical treatment of hemorrhoids: A critical appraisal of the current options. Arq Bras Cir Dig 27: 66-70, 2014.

4. Testa A and Torino G: Doppler-guided hemorrhoidal artery ligation (DG-HAL): A safe treatment of II-III degree hemorrhoids for all patients. Could it be potentially also good prophylaxis? Minerva Chir 65: 259-265, 2010.

5. Ratto C: THD Doppler procedure for hemorrhoids: The surgical technique. Tech Coloproctol 18: 291-298, 2014.

6. Gomez-Rosado JC, Sanchez-Ramirez M, Capitan-Morales LC, Valdes-Hernandez J, Reyes-Diaz ML, Cintas-Catena J, Guerrero-Garcia JM, Galan-Alvarez J and Oliva-Mompean F: One year follow-up after doppler-guided haemorrhoidal artery ligation. Cir Esp 90: 513-517, 2012 (In Spanish).
7. Huang H, Yao Y and Ding M: Clinical observation of blocking and fixing operation for mixed hemorrhoids with severe complications. Shanghai J Trad Chin Med 44: 45-46, 2010.

8. Ahmad A, Kant R and Gupta A: Comparative analysis ofdoppler guided hemorrhoidal artery ligation (DG-HAL) and infrared coagulation (IRC) in management of hemorrhoids. Indian J Surg 75: 274-277, 2013.

9. Lohsiriwat V: Approach to hemorrhoids. Curr Gastroenterol Rep 15: 332, 2013.

10. Buntzen S, Christensen P, Khalid A, Ljungmann K, Lindholt J, Lundby L, Walker LR, Raahave D and Qvist N; Danish Surgical Society: Diagnosis and treatment of haemorrhoids. Dan Med J 60: B4754, 2013.

11. Pol RA, van der Zwet WC, Hoornenborg D, Makkinga B, Kaijser M, Eeftinck Schattenkerk M and Eddes EH: Results of 244 consecutive patients with hemorrhoids treated with Doppler-guided hemorrhoidal artery ligation. Dig Surg 27: 279-284, 2010.

12. Wallis de Vries BM, van der Beek ES, de Wijkerslooth LR, van der Zwet WC, van der Hoeven JA, Eeftinck Schattenkerk M and Eddes EH: Treatment of grade 2 and 3 hemorrhoids with Doppler-guided hemorrhoidal artery ligation. Dig Surg 24: 436-440, 2007

13. Shi J, Jin J and Li J: Clinical observation on barron ligation and blocking and fixing operation in treatment of mixed hemorrhoids. J Liaoning Univ Trad Chin Med 16: 188-190, 2014.

14. Khafagy W, El Nakeeb A, Fouda E, Omar W, Elhak NG, Farid M and Elshobaky M: Conventional haemorrhoidectomy, stapled haemorrhoidectomy, Doppler guided haemorrhoidectomy artery ligation; post operative pain and anorectal manometric assessment. Hepatogastroenterology 56: 1010-1015, 2009.

15. 15.Zhang ZJ. effects of 'Tying, binding and fixing operation' in the treatment of mixed hemorrhoids: 40 cases reports. Chin Health Care and Nutrition 12: 7077-7077, 2013

16. Hall JF: Modern management of hemorrhoidal disease. Gastroenterol Clin North Am 42: 759-772, 2013.

17. Altomare DF and Giuratrabocchetta S: Conservative and surgical treatment of haemorrhoids. Nat Rev Gastroenterol Hepatol 10: 513-521, 2013.

18. Langley GB and Sheppeard H: The visual analogue scale: Its use in pain measurement. Rheumatol Int 5: 145-148, 1985.

19. Joshi GP and Neugebauer EA; PROSPECT Collaboration: Evidence-based management of pain after haemorrhoidectomy surgery. Br J Surg 97: 1155-1168, 2010.

20. Slauf P, Antoš F and Marx J: Complications of hemorrhoids. Rozhl Chir 93: 223-225, 2014 (In Czech).

21. Lienert M and Ulrich B: Doppler-guided ligation of the hemorrhoidal arteries. Report of experiences with 248 patients. Dtsch Med Wochenschr 129: 947-950, 2004 (In German).

22. Zhu J, Ding JH, Zhao K, Zhang B, Zhao Y, Tang HY and Zhao YJ: Complications after procedure for prolapse and hemorrhoids for circular hemorrhoids. Zhonghua Wei Chang Wai Ke Za Zhi 15: 1252-1255, 2012 (In Chinese). 